\title{
EMPREGO DA ANASTOMOSE PANCREATOJEJUNAL TIPO DUCTO-MUCOSA SEM CATETER TRANSANASTOMÓTICO EM PÂNCREAS DE CONSISTÊNCIA MOLE E DUCTO FINO: EXPERIÊNCIA INICIAL DO INSTITUTO NACIONAL DE CÂNCER
}

\author{
USE OF DUCT-TO-MUCOSA NONSTENTED PANCREATOJEJUNOSTOMY IN SOFT \\ PANCREAS AND SMALL PANCREATIC DUCT: INITIAL EXPERIENCE OF THE \\ NATIONAL CANCER INSTITUTE-BRAZIL
}

\author{
Jorge Mali Júnior, ACBC-PR 1; Gustavo S. Stoduto de Carvalho ${ }^{2}$; \\ Jurandir de Almeida Dias, ACBC-RJ ${ }^{3}$; Rafael de Oliveira Albagli, TCBC-RJ ${ }^{4}$
}

\begin{abstract}
RESUMO: Objetivo: O objetivo deste trabalho foi relatar a morbimortalidade associada à técnica de reconstrução pancreática utilizando anastomose pancreato-jejunal tipo ducto-mucosa, sem cateter transanastomótico, em pâncreas de consistência mole e ducto de Wirsung menor que três mm. Métodos: Analisamos consecutivamente os resultados de 16 pacientes submetidos à GDP com técnica de anastomose pancreato-jejunal tipo ducto-mucosa no Serviço de Cirurgia Abdômino-pélvica do INCA. Todos pacientes eram portadores de pâncreas de consistência mole e ducto pancreático fino $(<3 \mathrm{~mm})$. Resultados: Dos 16 pacientes analisados, oito eram do sexo masculino e com mediana de idade de 55 anos. No período pós-operatório um paciente apresentou fístula pancreática e houve um caso que evoluiu para óbito. Conclusão: Acreditamos que é possível a realização de anastomose pancreática tipo ductomucosa, sem cateter transanastomótico, em pacientes com ducto pancreático menor que três $\mathrm{mm}$ sem aumento da morbimortalidade (Rev. Col. Bras. Cir. 2007; 34(4): 218-221).
\end{abstract}

Descritores: Pancreaticoduodenectomia; Pancreaticojejunostomia; Anastomose cirúrgica/métodos; ducto pancreático.

\section{INTRODUÇÃO}

A gastroduodenopancreatectomia (GDP), já está bem estabelecida como a principal forma de tratamento para os tumores da região periampular. Este fato é baseado no acréscimo significativo de sobrevida que esta operação acarreta aos pacientes com tumores periampulares ressecáveis, quando comparado aos procedimentos paliativos.

Recentemente a indicação de GDP tem-se estendido aos chamados tumores não-periampulares (NPAP), que têm seus sítios primários mais comumente representados por colón, estômago, rim, bexiga, pele (melanoma) e pulmão. Estes tumores quando envolvem o bloco periampular, seja diretamente ou por metástase isolada, na ausência de doença à distância, tem na GDP a única chance de obter sobrevida a longo prazo.

Diferentemente da década de 70, atualmente a GDP apresenta taxas de mortalidade (0 - 5\%) e morbidade (20 $50 \%$ ) bastante aceitáveis, quando realizadas em centros de excelência em cirurgia pancreática ${ }^{1-3}$.

Dentre as complicações da GDP, a fístula pancreática continua sendo a mais importante, com uma incidência que varia de 5 a $25 \%$ nos grandes centros ${ }^{2-4}$. Vários fatores são relacionados à fístula pancreatojejunal: idade avança- da, tempo operatório, hemotransfusão per-operatória, nível de icterícia pré-operatória, consistência do pâncreas, calibre do ducto e experiência do cirurgião. Embora ainda não haja fatores etiológicos definidos para a fistula pancreática, a consistência do pâncreas e o calibre do ducto pancreático, associado à experiência do cirurgião, figuram entre as principais causas ${ }^{3,5,6}$.

De uma maneira geral, a reconstrução pancreatoentérica após GDP é causa de muitos debates em virtude das graves complicações que ela determina. Dentre os vários tipos de reconstruções pancreatoentéricas, a anastomose pancreatojejunal ducto-mucosa e a telescopagem são as mais utilizadas.

A consistência mole ou friável do pâncreas e o ducto de Wirsung de calibre fino configuram-se no maior desafio à habilidade do cirurgião pancreático na confecção da anastomose pancretaojejunal.

Neste contexto, há uma tendência atual na realização da anastomose tipo ducto-mucosa, principalmente em pâncreas de consistência endurecida e "ducto dilatado", ao contrário, quando o ducto é fino e o pâncreas de consistência mole ou friável dá-se preferência a anastomose tipo telescopagem ${ }^{3,6}$.

1. Cirurgião Oncológico do Instituto do Câncer de Londrina - PR.

2. Cirurgião do Hospital Naval Marcílio Dias, Rio de Janeiro - RJ.

3. Chefe do Serviço de Cirurgia Abdômino-Pélvica do INCA.

4. Coordenador do Grupo de Pâncreas do Serviço de Cirurgia Abdômino-Pélvica do INCA; Mestrando do Programa de Pós-Graduação da UFRJ.

Recebido em 04/12/2006

Aceito para publicação em 05/02/2007

Conflito de interesses: nenhum

Fonte de financiamento: nenhuma

Trabalho realizado na Seção de Cirurgia Abdômino - Pélvica do Instituto Nacional de Câncer (RJ) - Ministério da Saúde. 
Diante deste tópico - anastomose pancreatojejunal que é ainda considerado por muitos cirurgiões experientes o calcanhar de Aquiles da operação de Whipple, o Grupo de Pâncreas do Instituto Nacional de Câncer (INCA) avaliou a factibilidade da anastomose pancreatojejunal tipo ductomucosa, sem cateter transanastomótico, no pâncreas de consistência mole e ducto de Wirsung $<3 \mathrm{~mm}$. Os resultados preliminares deste estudo, no que tange à morbimortalidade operatória associada à técnica em questão, são relatados neste trabalho.

\section{MÉTODOS}

Foram analisados consecutivamente 16 pacientes submetidos à GDP com reconstrução pancreatoentérica utilizando a anastomose pancreatojejunal tipo ducto-mucosa, sem stent, em pâncreas de consistência mole e ducto de Wirsung fino. Este estudo foi realizado no Serviço de Cirurgia AbdôminoPélvica do INCA, no período de Abril 2000 de à Novembro de 2005, sendo todos os pacientes operados por uma mesma equipe de cirurgiões.

Foi definida como morbimortalidade operatória aquela que ocorreu dentro dos primeiros 30 dias após a cirurgia ou durante a mesma internação para a operação. Ducto pancreático fino foi considerado aquele com diâmetro menor que 3 $\mathrm{mm}$. A consistência do pâncreas era definida como mole, normal ou endurecida, de acordo com a experiência do cirurgião.

Dentre às várias definições de fístula pancreática pósoperatória decorrente de anastomose pancreatoentérica, utilizamos aquela que considera presença de fístula quando a dosagem de amilase no líquido peritoneal é igual ou maior três vezes o valor sérico a partir do terceiro dia de pós-operatório, independente da quantidade.

Quanto à técnica operatória, a anastomose pancreatojejunal tipo ducto-mucosa termino-lateral consistiu em secção de cinco cm da seromuscular da alça jejunal, expondo sua mucosa. Realizou-se uma sutura posterior unindo a camada seromuscular do jejuno com o parênquima pancreático, utilizando pontos separados de prolene 5.0. Realizou-se diérese de aproximadamente três $\mathrm{mm}$ da mucosa jejunal, anastomosando esta ao ducto pancreático através de quatro pontos cardeais de prolene 5.0. Finalmente procedeu-se a sutura anterior da camada seromuscular do jejuno com o parênquima pancreático. Durante a confecção da anastomose utilizou-se cateter de levine número seis, que foi retirado antes do término desta. Drenou-se a cavidade abdominal com dois drenos tubulares exteriorizados pelos hipocôncrios direito e esquerdo.

Quanto aos cuidados pós-operatórios, não foi utilizado de rotina medida farmacológica para prevenir a ocorrência de fístula. Iniciou-se dieta enteral através de sonda nasoentérica tão logo fosse possível.

\section{RESULTADOS}

Dos 16 pacientes analisados, oito eram do sexo masculino e oito do feminino, com mediana de idade de 55 anos ( 38-73 anos).
As indicações para a GDP foram devido à tumores não-periampulares na maioria dos casos ( 56\%): tumor de cólon = seis casos; tumor gástrico $=$ dois; tumor estromal de jejuno $=$ um caso. $\mathrm{O}$ restante dos doentes apresentavam tumores periampulares sendo; ampola de Vater $=$ três casos; pâncreas = um caso; terço distal de colédoco = um caso; duodeno $=$ um caso e tumor sólido-cístico de pâncreas $(\mathrm{Tu}$ mor de Frantz) $=$ um caso.

Histologicamente 14 casos deveram-se a adenocarcinoma, enquanto os outros dois eram GIST e tumor de Frantz, respectivamente.

A mediana do tempo operatório foi de 467 minutos (300-690 minutos), enquanto a de internação foi de 16 dias (1035 dias).

Dos 16 pacientes operados, cinco necessitaram de hemotransfusão intra-operatória, com mediana de volume sanguineo transfundido de $800 \mathrm{ml}$.

A taxa de fístula pancreatojejunal pós-operatória foi de $6 \%$ (um caso).

No período pós-operatório houve um caso que evoluiu para o óbito, decorrente de disfunção de múltiplos órgãos relacionada à fístula pancreática.

\section{DISCUSSÃO}

Recentemente a gastroduodenopancreatectomia (GDP) vem sendo realizada com mortalidade operatória menor que $5 \%$ em centros especializados ${ }^{1-3}$. Entretanto esta cirurgia é ainda associada a considerável morbidade, atingindo índices de até 50\%. A fístula pancreatojejunal sem dúvida alguma é sua principal complicação e a mais importante causa de óbito pós-opeartório, com taxas que variam de 5 a $25 \% \%^{2-4,7}$.

Quanto à reconstrução pancreática após GDP, ainda não há consenso de qual seja a melhor técnica de anastomose pancreatoentérica. ${ }^{8,9,10}$ No estudo prospectivo e randomizado de Stojadinovic et al., a taxa de fístula pancreática seguido de anastomose ducto-mucosa foi de $4 \%$ e após anastomose termino-terminal tipo telescopagem foi de $15 \%$, porém sem diferença estatística $(\mathrm{p}=0,09)^{11}$. Matsumoto et al. relataram que a taxa de fístula pancreática é influenciada pela técnica anastomótica, e que a técnica ducto-mucosa término-lateral tem uma incidência de fístula menor em relação a outros tipos de anastomose ${ }^{12}$. Segundo estudo realizado no INCA a anastomose pancreatojejunal tipo ducto-mucosa foi associada a taxa de fístula de $12 \%$, enquanto a telescopagem foi de $36 \%(\mathrm{p}=0,02)^{13}$. Pancreatogastroanastomose tem sido uma alternativa bastante utilizada em relação a pancreatojejunoanastomose, mas apesar de taxas de morbimortalidade semelhantes, o sangramento anastomótico tem limitado seu uso. ${ }^{11,12,14}$. Duffas et al. não mostraram diferença estatística no índice de fístula pancreática entre pancreatogastrostomia e pancreatojejunostomia ${ }^{5}$.

Dentre muitos fatores que podem influenciar a ocorrência de deiscência da anastomose pancreatojejunal, a consitência do pâncreas e o calibre do ducto pancreático estão fortemente associados à gênese da fístula pancreática. Yang et al., em 2005, mostraram numa análise multivariada que a textura do pâncreas $(\mathrm{p}=0,017)$ e o calibre do ducto $(\mathrm{p}=0,007)$ 
foram os únicos fatores de risco independentes associados com a fístula pancreática após GDP 6 . Neste mesmo estudo o índice de fístula pancreática da anastomose ducto-mucosa em relação à telescopagem foi menor (6,25\% VS 19,6\%), porém sem significância estatística.

Apesar de muita controvérsia acerca da técnica de anastomose pancreatojejunal, até poucos anos atrás existia um mito entre os cirurgiões pancreáticos: pacientes com ducto pancreático fino e pâncreas mole ou friável deveriam ser submetidos à anastomose tipo telescopagem. Suzuki et al. em 2002, propuseram que a seleção da técnica de anastomose pancretaojejunal deveria ser de acordo com o tamanho do ducto e a textura do pâncreas, sendo a anastomose tipo ductomucosa mais apropriada para pacientes com ducto pancreático largo (> 3mm) e pâncreas duro; enquanto que a anastomose convencional tipo telescopagem deveria ser empregada naqueles com pâncreas de textura mole e ducto fino ${ }^{3}$.

Recentemente, Duffas et al. num estudo multicêntrico controlado e randomizado, mostraram que entre outros fatores, a consistência normal do pâncreas e o diâmetro do ducto menor que $3 \mathrm{~mm}$ eram fatores de risco para fístula pancreática e/ou outras complicações intra-abdominais após GDP ${ }^{5}$.

No intuito de reduzir o índice de fístula pancreatoentérica, alguns autores advogam o uso de cateter transanastomótico principalmente nas anastomoses pancreatojejunais com ducto fino. Além disso, o emprego de cateteres transanastomóticos tem o objetivo de prevenir obstrução ductal e acelerar a cicatrização da anastomose pancreática, apesar de sua efetividade nunca ter sido comprovada ${ }^{15}$. Acreditamos que o uso, de rotina, de tubos através da anastomose pancreática seja dispensável porque o processo de cicatrização entre a mucosa jejunal e o ducto pancreático se faz no pós-operatório precoce, além do que o paciente requer cuidados mais complexos quanto maior o número de cateteres exteriorizados. Recentemente, Tani et al. não mostraram diferença significativa na taxa de fístula pancreática entre anastomose pancreatojejunal ducto-mucosa com e sem stents transanastomótico ${ }^{16}$

Atualmente acreditamos que a técnica empregada na reconstrução pancreática não parece ser o mais importante fator relacionado à fístula pancreática, ao passo que a experiência do cirurgião e a presença de fatores de risco (consistência do pâncreas e calibre do ducto) são os principais elementos associados à morbimortalidade .

Baseado nos nossos resultados preliminares, acreditamos que é possível a realização de anastomose pancreática tipo ducto-mucosa, sem cateter transanastomótico, em pacientes com ducto de Wirsung fino e pâncreas de consistência mole, sem aumento da morbimortalidade, em centros com experiência em cirurgia pancreática.

\begin{abstract}
Background: Our goal is to report the morbimortality associated to the pancreatic reconstruction technique using duct-to-mucosa nonstented pancreatojejunostomy in soft pancreas and Wirsung's duct less than 3 mm. Methods: We analyzed 16 consecutive patients submitted to DP with duct-to-mucosa pancreatojejunostomy technique in the Service of Abdominal-pelvic Surgery of NCI-Brazil. All patients had soft pancreatic texture and small pancreatic duct (< $3 \mathrm{~mm})$. Results: From those 16 patients, eight were males, with an mean age of 55 years old. In the post-operative period one patient developed pancreatic fistula. There had been one death. Conclusion: We believe that duct-tomucosa nonstented pancreatojejunostomy is possible in patients with pancreatic duct less than three mm and soft texture pancreas without increased morbimortality.
\end{abstract}

Key words: Pancreaticoduodenectomy; Pancreatojejunostomy; Anastomosis, Surgical/methods; Pancreatic ducts.

\section{REFERÊNCIAS}

1. Yeo CJ, Cameron JL, Sohn TA, Lillemoe KD, Pitt HA, Talamini MA, Hruban RH, Ord SE, Sauter PK, Coleman J, Zahurak ML, Grochow LB, Abrams RA. Six hundred fifty consecutive pancreaticoduodenectomies in the 1990s: pathology, complications, and outcomes. Ann Surg. 1997; 226(3):248-57; discussion 257-60.

2. Gouma DJ, van Geenen RCI, van Gulik TM, de Haan RJ, de Wit LT, Busch OR, Obertop H. Rates of complications and death after pancreaticoduodenectomy: risk factors and the impact of hospital volume. Ann Surg. 2000; 232(6):786-95.

3. Suzuki Y, Fujino Y, Tanioka Y, Hiraoka K, Takada M, Ajiki T, Takeyama Y, Ku Y, Kuroda Y. Selection of pancreaticojejunostomy techniques according to pancreatic texture and duct size. Arch Surg. 2002; 137(9):1044-7; discussion 1048.

4. van Berge HMI, de Wit LT, van Gulik TM, Obertop H, Gouma DJ. Incidence, risk factors, and treatment of pancreatic leakage after pancreaticoduodenectomy: drainage versus resection of the pancreatic remnant. J Am Coll Surg. 1997; 185(1):18-24.
5. Duffas JP, Sue B, Msika S, Fourtanier G, Muscari F, Hay JM, Fingerhut A, Millat B, Radovanowic A, Fagniez PL; French Associations for Research in Surgery. A controlled randomized multicenter trial of pancreatogastrostomy or pancreatojejunostomy after pancreatoduodenectomy. Am J Surg. 2005; 189(6):720-9.

6. Yang YM, Tian XD, Zhuang Y, Wang WM, Wan YL, Huang YT. Risk factors of pancreatic leakage after pancreaticoduodenectomy. World J Gastroenterol. 2005; 11(16):2456-61.

7. Balcom IV JH, Rattner DW, Warshaw AL, Chang Y, Fernandezdel Castillo C. Ten-year experience with 733 pancreatic resections: changing indications, older patients, and decreasing length of hospitalization. Arch Surg. 2001; 136(4):391-8.

8. Kakita A, Yoshida M, Takahashi T. History of pancreaticojejunostomy in pancreaticoduodenectomy: development of a more reliable anastomosis technique. $\mathbf{J}$ Hepatobiliary Pancreat Surg. 2001; 8(3):230-7.

9. Matsusue S, Takeda H, Nakamura Y, Nishimura S, Koizumi S. A prospective analysis of the factors influencing pancreaticojejunostomy performed using a single method, in 100 
consecutive pancreaticoduodenectomies. Surg Today. 1998; 28(7):719-26.

10. Z'graggen K, Uhl W, Friess H, Büchler MW. How to do a safe pancreatic anastomosis. J Hepatobiliary Pancreat Surg. 2002, 9(6):733-7.

11. Stojadinovic A, Brooks A, Hoos A, Jaques DP, Conlon KC, Brennan MF. An evidence-based approach to the surgical management of resectable pancreatic adenocarcinoma. J Am Coll Surg. 2003; 196(6):954-64.

12. Matsumoto Y, Fujii H, Miura K, Inoue S, Sekikawa T, Aoyama H, Ohnishi N, Sakai K, Suda K. Successful pancreatojejunal anastomosis for pancreatoduodenectomy. Surg Gynecol Obstet. 1992; 175(6):555-62.

13. Mali Jr J, Carvalho GS, Pierro G, Anghinoni M, Dias JA, Albagli R. Morbimortalidade relacionada à técnica de anastomose pancreática (ducto-mucosa x telescopagem) após cirurgia de Whipple. Rev Col Bras Cir. 2004; 31(1):74-7.

14. Yeo CJ, Cameron JL, Maher MM, Sauter PK, Zahurak ML, Talamini MA, Lillemoe KD, Pitt HA. A prospective randomized trial of pancreaticogastrostomy versus pancreaticojejunostomy after pancreaticoduodenectomy. Ann Surg. 1995; 222(4):580-8; discussion 588-92.
15. Biehl T, Traverso LW. Is stenting necessary for a successful pancreatic anastomosis? Am J Surg. 1992; 163(5):530-2.

16. Tani M, Onishi H, Kinoshita H, Kawai M, Ueno M, Hama T, Uchiyama K, Yamaue H. The evaluation of duct-to-mucosal pancreaticojejunostomy in pancreaticoduodenectomy. World J Surg. 2005; 29(1):76-9.

Como citar este artigo:

Mali Jr J, Carvalho GS, Dias JA, Albagli RO. Emprego da anastomose pancreatojejunal tipo ducto-mucosa sem cateter transanastomótico em pâncreas de consistência mole e ducto fino: experiência inicial do Instituto Nacional de Câncer (INCa). Rev Col Bras Cir. 2007; 34(4). Disponível em URL: www.scielo.br/br

Endereço para correspondência:

Rafael Albagli

Rua Muniz Barreto, 396 / AP: 1002

Cep: 22251-090

Botafogo- RJ

rafaelalbagli@uol.com.br 